\title{
Testing LISA drag-free control with the LISA technology package flight experiment
}

\author{
D Bortoluzzi ${ }^{1}$, P Bosetti $^{1}$, L Carbone $^{2}$, A Cavalleri $^{2}$, A Ciccolella $^{3}$, \\ M Da Lio ${ }^{1}$, K Danzmann ${ }^{4,5}$, R Dolesi ${ }^{2}$, A Gianolio ${ }^{3}$, G Heinzel $^{4,5}$, \\ D Hoyland $^{6}$, C D Hoyle ${ }^{2}$, M Hueller ${ }^{2}$, F Nappo ${ }^{7}$, M Sallusti ${ }^{3}$, P Sarra ${ }^{7}$, \\ M Te Plate ${ }^{3}$, C Tirabassi ${ }^{3}, \mathbf{S}$ Vitale $^{2}$ and W J Weber ${ }^{2,8}$ \\ ${ }^{1}$ Dipartimento di Ingegneria, Università di Trento, Trento, Italy \\ 2 Dipartimento di Fisica and INFN, Università di Trento, Trento, Italy \\ ${ }^{3}$ ESA-ESTEC, Nordwijk, The Netherlands \\ ${ }^{4}$ Max-Planck Institut für Gravitationsphysik, Albert-Einstein-Institut, Hannover, Germany \\ ${ }^{5}$ Universität Hannover, Hannover, Germany \\ ${ }^{6}$ Department of Physics and Astronomy, University of Birmingham, UK \\ ${ }^{7}$ Carlo Gavazzi Space S.p.A., Milano, Italy \\ E-mail: weber@science.unitn.it
}

Received 13 December 2002

Published 28 April 2003

Online at stacks.iop.org/CQG/20/S89

\begin{abstract}
The LISA test masses must be kept free of stray acceleration noise to within $3 \times 10^{-15} \mathrm{~m} \mathrm{~s}^{-2} \mathrm{~Hz}^{-1 / 2}$ in order to obtain the low-frequency gravitational wave sensitivity goal. The LISA technology package (LTP) is a dedicated ESA flight experiment for testing the drag-free control technology that must ensure purity of free fall in the LISA mission. We present here a brief description of the LTP experimental configuration, specific measurements to be performed and the requirements that must be met in order to demonstrate the LTP stray acceleration upper limit goal of $3 \times 10^{-14} \mathrm{~m} \mathrm{~s}^{-2} \mathrm{~Hz}^{-1 / 2}$ at $1 \mathrm{mHz}$.
\end{abstract}

PACS number: $04.80 . \mathrm{Nn}$

\section{Introduction: drag-free control for LISA and LTP}

The LISA gravitational wave sensitivity will be limited at low frequencies by stray force contamination of the nominally free falling orbits of the test masses serving as the LISA interferometer end mirrors. Conceptually, a drag-free control system to protect the purity of the test mass free fall consists of a satellite, of mass $M$, shielding a floating test mass (mass $m$ ), and employing high-precision thrusters to centre itself about the test mass based on

8 Author to whom correspondence should be addressed. W J Weber, Dipartimento di Fisica, Università di Trento, Via Sommarive 14, 38050 Povo (TN), Italy. 
Table 1. Requirements for LISA and LTP $[1,2]$

\begin{tabular}{lllll}
\hline & \multicolumn{3}{l}{ LISA } & LTP \\
& $0.1 \mathrm{mHz}$ & $1 \mathrm{mHz}$ & \\
\hline Acceleration noise & $S_{a_{n}}^{1 / 2}$ & 3 & 30 & $\mathrm{fm} \mathrm{s}^{-2} \mathrm{~Hz}^{-1 / 2}$ \\
Position sensor noise & $S_{x_{n}}^{1 / 2}$ & 1.8 & 1.8 & $\mathrm{~nm} \mathrm{~Hz}^{-1 / 2}$ \\
Spacecraft position noise & $\frac{F_{\mathrm{ext}}}{M \omega_{\mathrm{DF}}^{2}}$ & 1.8 & 4.7 & $\mathrm{~nm} \mathrm{~Hz}^{-1 / 2}$ \\
Parasitic stiffness & $\left|\omega_{p}^{2}\right|$ & 4 & 20 & $\times 10^{-7} \mathrm{~s}^{-2}$ \\
Compensated dc acceleration & $\Delta a_{\mathrm{dc} x}$ & $\cdots$ & 1.3 & $\mathrm{~nm} \mathrm{~s}^{-2}$ \\
\hline
\end{tabular}

feedback from a relative position sensor (also known as a 'gravitational sensor,' as it measures the satellite position with respect to a geodetic reference). The resulting residual test mass acceleration noise will depend on the stray forces $f_{\text {str }}$, introduced by the imperfect shielding of environmental disturbances and by the satellite itself. Additionally, noisy spacecraft motion, caused by position sensing noise, $x_{n}$, and the finite gain of the drag-free control loop to external forces $F_{\text {ext }}$ acting on the spacecraft, will couple to the test mass via any steady force gradient, or parasitic stiffness, characterized by spring constant $k_{p}$. The closed loop residual acceleration is given by

$$
a_{n}=\frac{f_{\mathrm{str}}}{m}+\omega_{p}^{2}\left(x_{n}+\frac{F_{\mathrm{str}}}{M \omega_{\mathrm{DF}}^{2}}\right),
$$

where $M \omega_{\mathrm{DF}}^{2}$ is the drag-free force to displacement gain, and $\omega_{p}^{2} \equiv \frac{k_{p}}{m}$. Equation (1) is valid in the limit of high drag-free gain, such that $\omega_{\mathrm{DF}}^{2} \gg \omega_{p}^{2}$ and $\omega_{\mathrm{DF}}^{2} \gg \omega^{2}$.

The LISA low-frequency sensitivity goal at low frequencies [1], extending down to $0.1 \mathrm{mHz}$, requires a limit of the test mass acceleration noise

$$
S_{a_{n}}^{1 / 2}=\sqrt{\frac{S_{f_{\mathrm{str}}}}{m^{2}}+\left|\omega_{p}^{4}\right|\left(S_{x_{n}}+\frac{S_{F_{\mathrm{str}}}}{M^{2} \omega_{\mathrm{DF}}^{4}}\right)} \leqslant 3 \times 10^{-15} \mathrm{~m} \mathrm{~s}^{-2} \mathrm{~Hz}^{-1 / 2} .
$$

We note that test mass acceleration noise converts to an effective gravitational wave strain measurement noise proportional to $\frac{S_{a_{n}}^{1 / 2}}{\omega^{2}}$. Reaching the $3 \mathrm{fm} \mathrm{s}^{-2} \mathrm{~Hz}^{-1 / 2}$ goal in equation (2) means that acceleration noise would become the dominant noise source limiting the LISA sensitivity at frequencies below a few $\mathrm{mHz}$, where it climbs above the interferometric measurement shot noise limit [1]. Larger acceleration noise levels would reduce the lowfrequency sensitivity and cut into the low-frequency side of the band in which the gravitational wave sensitivity is photon limited.

A preliminary apportioning of the acceleration noise 'budget' in equation (2) gives $1 \mathrm{fm} \mathrm{s}^{-2} \mathrm{~Hz}^{-1 / 2}$ to the coupling term, requiring spacecraft control at the several $\mathrm{nm} \mathrm{Hz}^{-1 / 2}$ level and parasitic stiffness frequencies $\frac{\left|\omega_{p}\right|}{2 \pi}$ kept below $0.1 \mathrm{mHz}$ (see table 1). The bulk of the noise budget is left to stray forces $f_{\text {str. }}{ }^{9}$

The level of isolation from stray forces required by equation (2), which corresponds to $6 \mathrm{fN} \mathrm{Hz}^{-1 / 2}$ for $2 \mathrm{~kg}$ test masses, is a substantial leap from the demonstrated performance of previous drag-free flight experiments [3] and of sensitive earth-based weak force experiments [4]. As such, the LISA technology package (LTP), a dedicated space experiment to demonstrate LISA drag-free control, is being prepared by the European Space Agency and is scheduled

9 While the true acceleration noise requirements increase as $f^{2}$ above $3 \mathrm{mHz}$ [2], for simplicity, we cite here, in equation (2) and table 1, only the more stringent low-frequency limit. 


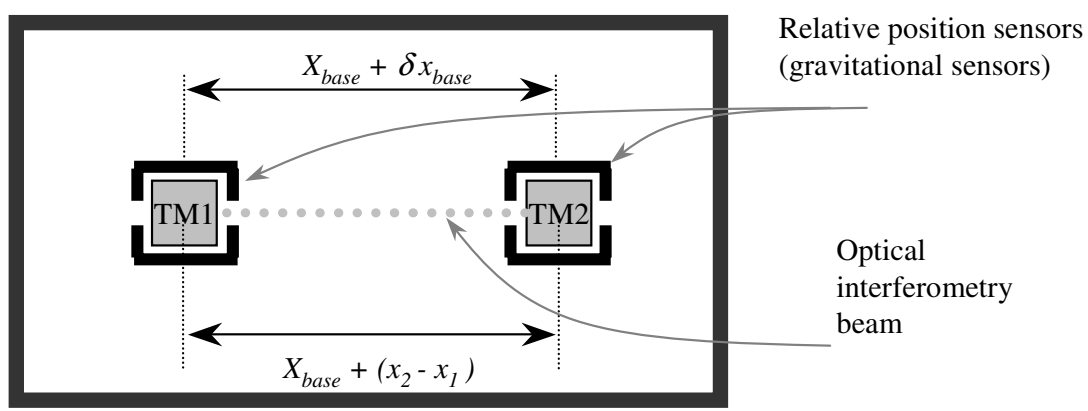

Figure 1. Cartoon of the LTP experiment. $X_{\text {base }} \approx 30 \mathrm{~cm}, \delta x$ is the baseline distortion between the two position sensors (black), and $\Delta x_{12} \equiv x_{2}-x_{1}$ is the differential test mass displacement to be measured interferometrically.

for a 2006 launch aboard the SMART-2 mission [5]. A parallel effort in the United States will participate on the same flight with a similar experiment, called the disturbance reduction system (DRS).

\section{LTP experimental configuration}

LTP, illustrated in figure 1, compresses a single arm of the triangular LISA interferometer into one spacecraft. The goal is to demonstrate drag-free flight at the level of $3 \times 10^{-14} \mathrm{~m} \mathrm{~s}^{-2} \mathrm{~Hz}^{-1 / 2}$ between 1 and $30 \mathrm{mHz}$. Achievement of this goal, a factor 10 above the LISA acceleration noise requirement, represents both true flight test of drag-free control and significant improvement from ground-based measurements, and is still feasible within the simplified single spacecraft LTP experimental configuration. Considering the 'red' nature of many key noise sources $[2,6,7]$, the LTP goal is also relaxed in its official lower frequency limit of $1 \mathrm{mHz}$, a factor 10 above the nominal LISA low-frequency limit of $0.1 \mathrm{mHz}$. The experimental apparatus consists of a spacecraft equipped with precision ' $\mu \mathrm{N}$ ' thrusters and two electrostatic position sensors placed roughly $30 \mathrm{~cm}$ apart and each hosting a flying test mass (TM1 and TM2). The relative displacement of the two test masses, $\Delta x_{12} \equiv x_{2}-x_{1}$, is measured interferometrically and serves as the main probe of the drag-free performance.

The spacecraft cannot simultaneously follow both masses, and so the trajectory of only one test mass, or an average of the two test mass positions, will serve as the drag-free reference along the $x$ or measurement axis. To prevent the trajectories of the two masses from diverging in response to any differential force, at least one mass must have a 'suspension' forcing it to follow the spacecraft. This is not true in LISA, where the satellite can simultaneously follow two masses without applying forces along their two sensitive axes, defined by the two legs of the interferometry triangle [5]. In both LISA and LTP, forces and torques must be applied on all non-measurement degrees of freedom, an important consideration for cross-talking concerns.

Six degree-of-freedom capacitive position sensors [6, 7] will measure the relative test mass-satellite displacements, which are referred to, along the $x$ axis, as $\Delta x_{1}\left(\equiv x_{1}-x_{S C}\right)$ and $\Delta x_{2}\left(\equiv x_{2}-x_{S C}\right)$. The same electrodes used for position sensing will also apply the electrostatic actuation forces needed to suspend at least one test mass, with frequency dependent force to displacement gain $\omega_{E S}^{2}(\omega)$. The electrostatic suspension must (1) compensate for imbalance $\Delta a_{\mathrm{dc}}$ in the residual dc acceleration between TM1 and TM2, likely dominated by the spacecraft gravitational field, and (2) compensate the likely negative (and thus unstable) net parasitic coupling of the test mass to the satellite $\left(\omega_{p}^{2}<0\right)$, dominated by the electric fields applied 
for the position sensor readout. An optimized control law, providing both high gain at low frequencies and the minimum in-band coupling needed to stabilize the negative spring, has been found, with gain $\omega_{E S}^{2} \approx 2\left|\omega_{p}^{2}\right|$ across the LTP measurement band [8].

Electrostatic actuation adds both force noise and, as the applied forces depend on position, a significant contribution to the negative parasitic stiffness $\left(\approx \frac{-2 \Delta a_{\mathrm{dc}}}{d}\right.$, where $d$ is the distance between the test mass surface and the actuation electrodes, $4 \mathrm{~mm}$ in the current design). The magnitudes of the additional noise and stiffness will increase with the levels of both the dc force and parasitic stiffness to be compensated. As such, in LTP we design for a maximum allowable dc acceleration imbalance $\Delta a_{\mathrm{dc}}$, and establish a corresponding increased allowance for the parasitic stiffness. This need to apply actuation forces along the measurement axis in LTP is thus an important, and performance limiting, departure from the LISA drag-free scheme. However, following the apportioning in table 1 will still allow demonstration of dragfree flight to within a factor 10 of the LISA goal, providing a feasible and representative flight test of LISA position sensors, thrusters and control laws in a single spacecraft experiment.

Beyond the baselined configuration of capacitive position sensors and an interferometric measurement of $\Delta x_{12}$, the optical metrology bench [9] will contain the additional optics and detectors to allow interferometric readout of $\Delta x_{1}$. Both interferometry detectors will also be made from quadrant photodiodes, which will allow optical readout of the angular orientations of the test mass reflecting surface, differentially between the two test masses and of TM1 with respect to the spacecraft. These additional optical readouts open the possibility of testing optical position sensors for the drag-free feedback on different degrees of freedom and of a lower noise readout of $\Delta x_{1}$ for specific tests.

\section{LTP operational modes and planned experiments}

\subsection{Primary control scheme and acceleration noise measurement}

In the primary test of LTP [2], the spacecraft drag-free control will be driven by the readout of the TM1 position sensor (with drag-free gain $\omega_{\mathrm{DF}}^{2}$ ), and the second mass will be controlled electrostatically (with gain $\omega_{E S}^{2}$ ) to follow the satellite, according to the TM2 position sensor. The resulting optical measurement of $\Delta x_{12}$ can be viewed as a probe of the drag-free performance of TM1, involving the force noise $f_{1}$, position sensor noise $x_{1 n}$, stiffness $\omega_{1 p}^{2}$ and the satellite jitter $\frac{F_{\mathrm{str}}}{M \omega_{\mathrm{DF}}^{2}}$. However, the differential, or gradiometer, LTP signal readout also reflects, in a quantitatively significant way, the properties of the electrostatically controlled second test mass, $f_{2}, x_{2 n}$ and $\omega_{2 p}^{2}$. This is seen in the closed loop transfer function, which also includes the additive optical measurement noise $x_{n \text {,opt }}$ and possible distortion of the baseline separating the two position sensors, $\delta x$ :

$$
\begin{aligned}
\Delta x_{\mathrm{opt}}=\Delta x_{12} & +x_{n, \mathrm{opt}}=\frac{1}{\omega^{2}-\left(\omega_{2 p}^{2}+\omega_{E S}^{2}\right)}\left[\frac{f_{1}-f_{2}}{m}+\left(x_{1 n}+\frac{F_{\mathrm{str}}}{M \omega_{\mathrm{DF}}^{2}}\right)\right. \\
& \left.\times\left[\omega_{1 p}^{2}-\left(\omega_{2 p}^{2}+\omega_{E S}^{2}\right)\right]+x_{2 n} \omega_{E S}^{2}-\delta x\left(\omega_{2 p}^{2}+\omega_{E S}^{2}\right)\right]+x_{n, \mathrm{opt}} .
\end{aligned}
$$

The gradiometer configuration thus results in a signal sensitive only to the difference in stray forces $\left(f_{2}-f_{1}\right)$ and coupling $\left(\left[\omega_{1 p}^{2}-\left(\omega_{2 p}^{2}+\omega_{E S}^{2}\right)\right]\right)$ acting on the two test masses. For the stray forces, however, the most dangerous sources are short-range electrostatic and thermal noise effects, which are likely to be uncorrelated between the two sensors. Additionally, for electrostatic actuation gain chosen to have $\omega_{E S}^{2} \approx 2\left|\omega_{2 p}^{2}\right|$ and $\omega_{1 p}^{2} \approx \omega_{2 p}^{2} \equiv \omega_{p}^{2}$, the differential stiffness $\left[\omega_{1 p}^{2}-\left(\omega_{2 p}^{2}+\omega_{E S}^{2}\right)\right] \approx 2 \omega_{p}^{2}$, coupling the relative test mass-satellite motion into the interferometry signal. Measurements of this stiffness will be discussed in section 3.1.2. 
While stiffness and stray force contribute in a quantitatively different manner than aboard LISA, the measured noise in the optical signal (equation (3)) still allows placement of an upper limit on the total stray acceleration noise of a drag-free test mass, $S_{a_{n}}^{1 / 2}$. Assuming uncorrelated and equal magnitude in the force and position sensor noise for TM1 and TM2 $\left(S_{f_{\mathrm{str}}}\right.$ and $\left.S_{x_{n}}\right)$ and, additionally, equal parasitic stiffness $\left(\omega_{p}^{2}\right)$,

$S_{\Delta x_{\mathrm{opt}}}^{1 / 2} \geqslant \frac{1}{\omega^{2}-\left(\omega_{2 p}^{2}+\omega_{E S}^{2}\right)} \sqrt{\frac{2 S_{f}}{m^{2}}+4\left|\omega_{p}^{4}\right|\left(2 S_{x}+\frac{S_{F}}{M^{2} \omega_{\mathrm{DF}}^{4}}\right)}>\frac{1}{\omega^{2}-\left(\omega_{2 p}^{2}+\omega_{E S}^{2}\right)} \sqrt{2} S_{a}^{1 / 2}$.

In order to demonstrate the acceleration noise limit goal of $S_{a_{n}}^{1 / 2}<30 \mathrm{fm} \mathrm{s}^{-2} \mathrm{~Hz}^{-1 / 2}$, the closed loop optical noise must satisfy $S_{\Delta x_{\mathrm{opt}}}^{1 / 2}<1 \mathrm{~nm} \mathrm{~Hz}^{-1 / 2}$ at $1 \mathrm{mHz}$. Note that this is a worst-case acceleration noise upper limit involving an exaggerated role of the stiffness and electrostatic feedback with respect to the performance aboard LISA.

To unambiguously characterize the acceleration noise, the optical noise $x_{n, \text { opt }}$ and optical bench baseline distortion $\delta x$ should be designed to stay below the $0.1 \mathrm{~nm} \mathrm{~Hz}{ }^{-1 / 2}$ level at $1 \mathrm{mHz}$. This should be attainable with the envisioned heterodyne optical interferometer and high-stability ULE glass optical bench [9].

Additional information lies in the simultaneously monitored TM1 sensor signal, $\Delta x_{1 s} \equiv$ $\Delta x_{1}+x_{1 n}$. For high, but finite, drag-free control gain, the thrusters will, in trying to zero $\Delta x_{1 s}$, very nearly compensate all sensor noise and stray forces acting on the test mass. The closed loop noise in the sensor output is then dominated by the residual jitter of the spacecraft in response to external forces,

$$
S_{\Delta x_{1 s}}^{1 / 2} \approx \frac{S_{F_{\mathrm{str}}}^{1 / 2}}{M \omega_{\mathrm{DF}}^{2}}
$$

This allows a separation of the external force noise contribution from the total acceleration noise measured in equation (4). Note also that the external forces $F_{\text {str }}$ acting on the satellite include the force noise of the thrusters.

An alternate approach to this primary test is to use the optical readout of $\Delta x_{1}$ for controlling the satellite, which will decrease the position noise and allow a test of drag-free control using optical sensing. The higher resolution optical readout of $\Delta x_{1}$ also allows direct measurement of the TM1 sensor noise, $S_{x_{1 n}}^{1 / 2}$.

3.1.1. Coherent force detection. In this same control scheme, a modulated force $f_{0} \sin \omega_{0} t$ applied to TM1 will produce a coherent differential motion with amplitude

$$
\Delta x_{12} \approx \Delta x_{2} \approx \frac{f_{0}}{m} \frac{1}{\omega_{0}^{2}-\left(\omega_{2 p}^{2}+\omega_{E S}^{2}\right)} .
$$

Note that application of the force to TM2 instead of TM1 would simply add a negative sign to equation (6). The force resolution attained from the laser signal $\Delta x_{\mathrm{opt}}$, in a measurement time $T$, should be limited by the stray acceleration limit discussed in the previous section, or

$$
\Delta\left(f_{0}\right) \approx \sqrt{2} m \sqrt{\frac{2 S_{a_{n}}}{T}} \approx 2 \mathrm{fN} \times\left(\frac{S_{a_{n}}^{1 / 2}}{30 \mathrm{fm} \mathrm{s}^{-2} \mathrm{~Hz}^{-1 / 2}}\right)\left(\frac{1 \mathrm{~h}}{T}\right)^{1 / 2} .
$$

A number of applications of such force modulation, with resolution at the fN level possible in a $1 \mathrm{~h}$ measurement, are envisioned for LTP.

- Calibration. Modulated application of a known force allows calibration of the differential transfer function appearing as the denominator of equations (3) and (6). An electrostatic 
force could be known, by electrostatic modelling and on-ground measurement, at the $1 \%$ level. This calibrates the other force measurements, including the stiffness measurements discussed in the next section.

- Charge and dc bias measurement. Low-frequency voltages applied with the actuation circuitry will allow measurement of the accumulated test mass electric charge and serve as the feedback error signal for the charge management system, which will use UV light and electrostatic biasing to maintain, either periodically or continuously, the test mass charge close to zero $[10,11]$. Another combination of modulated electrode voltages can allow measurement, and possible balancing, of the stray dc biases on the electrodes in the position sensors, which can be an important noise source because of coupling to random test mass charging and low-frequency dielectric noise.

- Disturbance feedthrough. In addition to electrostatic disturbances, coherent modulation of temperature gradients and magnetic fields would allow characterization of the force 'feedthrough' of these disturbances which couple, respectively, to outgassing and radiation effects and magnetic impurity.

3.1.2. Stiffness measurement. In this control scheme, the differential stiffness $\left[\omega_{1 p}^{2}-\left(\omega_{2 p}^{2}+\right.\right.$ $\left.\left.\omega_{E S}^{2}\right)\right]$ can be measured by controlling the satellite to a slowly oscillating setpoint, rather than the TM1 position readout zero. For a modulated drag-free setpoint $x_{0} \cos \omega_{0} t$, the differential motion in $\Delta x_{12}$ has amplitude

$$
\Delta x_{12} \approx-x_{0} \frac{\omega_{1 p}^{2}-\left(\omega_{2 p}^{2}+\omega_{E S}^{2}\right)}{\omega_{0}^{2}-\left(\omega_{2 p}^{2}+\omega_{E S}^{2}\right)} .
$$

The resultant differential stiffness resolution, limited by acceleration noise, is calculated as in equation (7) and given by

$$
\Delta\left(\omega^{2}\right) \approx 4 \times 10^{-8} \mathrm{~s}^{-2} \times\left(\frac{25 \mathrm{~nm}}{x_{0}}\right)\left(\frac{S_{a_{n}}^{1 / 2}}{30 \mathrm{fm} \mathrm{s}^{-2} \mathrm{~Hz}^{-1 / 2}}\right)\left(\frac{1 \mathrm{~h}}{T}\right)^{1 / 2} .
$$

This is a $2 \%$ measurement of the differential stiffness projected for LTP (10\% for LISA), which can be improved for larger setpoint modulation amplitude. If there is a significant stochastic source of stiffness, due to unmodelled short-range electrostatic or magnetic forces in the position sensors, this measurement should detect it.

Modulating the drag-free setpoint also will excite a signal in $\Delta x_{2}$, detectable by the TM2 relative position sensor as a measure of the absolute parasitic stiffness $\omega_{1 p}^{2}$,

$$
\Delta x_{2} \approx-x_{0} \frac{\omega_{0}^{2}-\omega_{1 p}^{2}}{\omega_{0}^{2}-\left(\omega_{2 p}^{2}+\omega_{E S}^{2}\right)} .
$$

The estimate of $\omega_{1 p}^{2}$ extracted from equation (10) is likely to be limited by the accuracy of the calibration of the denominator, as $\omega_{1 p}^{2}$ will be of an order 20 times smaller than $\omega_{0}^{2}$ even for slow modulation at $1 \mathrm{mHz}$. For a $1 \%$ force calibration, as discussed in section 3.1.1, the measurement in equation (10) will yield a measurement of $\omega_{1 p}^{2}$ at the $20 \%$ level. Though not a high-precision measurement, it is still a useful probe for any unmodelled phenomenon that could produce stiffness at significant levels.

\subsection{Control using laser interferometry signal}

In an alternate control scheme, TM2 will be controlled electrostatically to null the differential displacement interferometry signal $\Delta x_{\text {opt }}$ (rather than the TM2 position sensor), with the TM1 
position sensor still used as the reference for the spacecraft drag-free control. 'Locking' the second mass directly to the first in this manner presents several advantages that are evident in the closed loop $\Delta x_{12}$ signal:

$$
\begin{aligned}
& \Delta x_{\mathrm{opt}}=\frac{1}{\omega^{2}-\left(\omega_{2 p}^{2}+\omega_{E S}^{2}\right)}\left[\frac{f_{1}-f_{2}}{m}+\left(x_{1 n}+\frac{F_{\mathrm{str}}}{M \omega_{\mathrm{DF}}^{2}}\right)\left[\omega_{1 p}^{2}-\omega_{2 p}^{2}\right]\right. \\
& \left.-\delta x \omega_{p 2}^{2}+x_{n, \text { opt }}\left(\omega^{2}-\omega_{2 p}^{2}\right)\right] \text {. }
\end{aligned}
$$

This scheme offers several important advantages. The laser signal $\left(x_{n, \text { opt }}\right)$ should be quieter than the capacitive position sensor $\left(x_{2 n}\right)$ by a factor 20 , which in this configuration allows a much more precise control of TM2. Additionally, the relative position noise of TM1 and the satellite now couples to the measurement signal through $\left(\omega_{1 p}^{2}-\omega_{2 p}^{2}\right)$. This differential stiffness, nominally zero, can be measured by modulating the setpoint, in analogy to section 3.1.2, and then tuned to zero by application of small ac electrical fields. Cancelling this stiffness at the $1 \%$ level would reduce the coupling term in equation (11) by a factor 100 , allowing a very sensitive probe of the now isolated differential force, $\left(f_{2}-f_{1}\right)$. An extraction of $S_{f}^{1 / 2}$ from the measurement of the noise in $\Delta x_{\mathrm{opt}}$, given in this configuration of zero coupling by

$$
S_{\Delta x_{\mathrm{opt}}}^{1 / 2} \geqslant \frac{1}{\omega^{2}-\left(\omega_{2 p}^{2}+\omega_{E S}^{2}\right)} \frac{\sqrt{2} S_{f}^{1 / 2}}{m},
$$

could be limited by the interferometry noise, $x_{n, \text { opt }}$ at levels very near to the LISA total stray-force goal of several $\mathrm{fN} \mathrm{Hz}^{-1 / 2}$.

\section{LTP system implementation}

A great deal of analysis is needed to translate the LTP scientific objective, the demonstration of drag-free flight within a factor 10 of the LISA goal, into a series of engineering requirements and flight hardware plans for the LTP apparatus. From the first step of decomposing the acceleration noise budget into random forces and coupling of relative test mass-satellite motion, illustrated in equation (3) and table 1, flows a series of derived requirements, addressing the individual instruments' performance as well as their collective, closed loop disturbance. As an example, the interferometry bench must be analysed not only for its measurement sensitivity, but also for its mass distribution, which creates a dc gravitational force on the test masses, which in turn demands higher actuation voltages, which increases the parasitic coupling and thus results in an amplified acceleration noise caused by the capacitive sensor's noise and the thruster force noise. Such distributed contributions of disturbance to the primary science objectives of LTP demand a system level or 'architectural' design analysis that goes beyond standard interfacing between instruments.

A preliminary design of the LTP apparatus is shown in figure 2. The key instruments of the LTP, consisting of the electrostatic position sensor [6, 7] (including a charge management system $[10,11]$ and mechanical caging system to secure the test mass during launch and, when necessary, in flight [12]), optical bench and interferometry hardware [9], and $\mu \mathrm{N}$ thrusters [13], and their contributions to the overall acceleration noise, are all described in detail in other papers in this symposium. As such, we will not discuss any of these instruments in detail here, but, rather, mention several key disturbance sources that impact on the LTP 


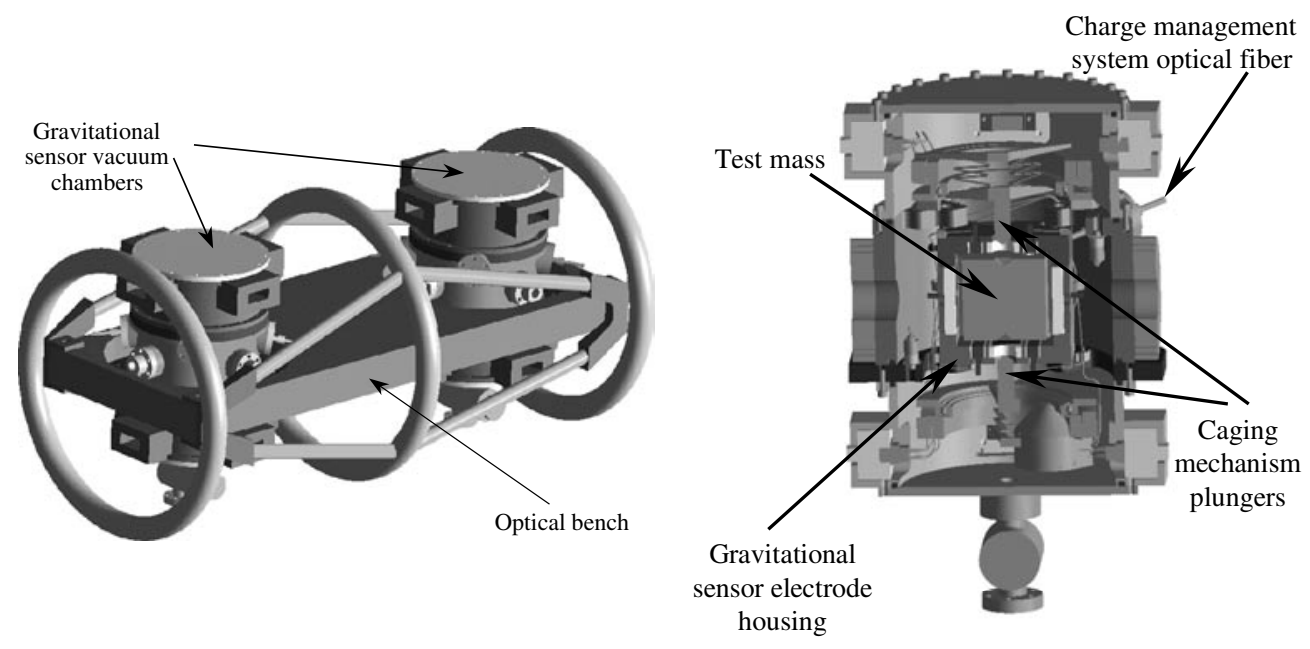

Figure 2. Preliminary schematic design of the LTP apparatus, shown here on the left without its cylindrical thermal shield. An enlargement of the gravitational sensor vacuum chamber and its key subsystems is shown on the right.

design in multiple places. A full description of these and other design-driving considerations is found in [2].

- Magnetic. Magnetic field interaction with the test-mass magnetic moment contributes several important sources of force noise. While the envisioned $\mathrm{Au} / \mathrm{Pt}$ alloy should have very low susceptibility, the presence of ferromagnetic impurities could give a significant permanent moment, assumed cautiously (see [14]) to be of the order $\approx 3 \times 10^{-7} \mathrm{~A} \mathrm{~m}^{2}$. In this case, the dominant magnetic disturbance, or, alternatively, the most challenging magnetic requirement, comes from the interaction with the field gradient, $f_{x}=\vec{m}_{0} \cdot(\nabla \vec{B})_{x}$. To keep this noise source at the $5 \mathrm{fm} \mathrm{s}^{-2} \mathrm{~Hz}^{-1 / 2}$ level, the field gradient fluctuations at the test mass must be less than $25 \mathrm{nT} \mathrm{m}^{-1} \mathrm{~Hz}^{-1 / 2}$. This is primarily a constraint on the stability of magnetic field sources placed close to the test masses.

- Gravitational. Gravitational imbalance of the entire spacecraft is assumed to dominate the LTP budget for the differential dc acceleration $\Delta a_{\mathrm{dc}}$. The gravitational acceleration of TM2 in the field of the TM1 sensor and vacuum chamber is itself several times the $1.3 \mathrm{~nm} \mathrm{~s}^{-2}$ level. As such, compensation masses and a rather precise mass distribution analysis are essential. For sources located on the outer edges of the spacecraft, the differential gravitational acceleration is suppressed well below the common mode, allowing, for example, an electronics box of mass $1 \mathrm{~kg}$ to be characterized as a point mass, with $2 \mathrm{~mm}$ and $50 \mathrm{gm}$ uncertainties in the estimate of its placement and mass. The optical bench, however, must be mounted with $200 \mu \mathrm{m}$ precision, with the mass distribution calculated at the $1 \%$ level in $5 \mathrm{~mm}$ cubic grid volumes.

- Thermal and vacuum. Radiation pressure differences, radiometric effects, and potentially temperature dependent outgassing effects demand that the test mass see a very uniform and stable thermal environment [7]. The temperature difference $\Delta T$ between the surfaces facing opposite sides of the test mass must fluctuate less than $10^{-4} \mathrm{~K} \mathrm{~Hz}^{-1 / 2}$. The current sensor design [7] employs a high thermal conductivity metallic/ceramic construction to suppress thermal gradients introduced by power consumption on the optical bench and for passive thermal spacecraft shielding from external heat loads. 


\section{Conclusion}

The measurements described here do not exhaust the list of planned tests aboard LTP, but should represent the ability of the LTP experiment to characterize the drag-free performance relevant to LISA. In addition to an upper limit on the total acceleration noise, the design allows a high-resolution measurement of the parasitic stiffness, position noise and stray forces, as well as characterization of a number of specific disturbances. Finally, the tested hardware (position sensors, thrusters and charge-management systems), as well as the drag-free and electrostatic suspension control laws, are designed to LISA specifications, making LTP a truly representative bridge between ground-based testing and the final gravity-wave mission.

\section{References}

[1] LISA Study Team 1998 LISA: a cornerstone mission for the observation of gravitational waves ESA-SCI(2000)11 System and Technology Study Report. LISA Pre-Phase A Report webpage http://lisa.jpl.nasa.gov

[2] Vitale S 2002 LISA Technology Package Architect Final Report ESTEC contract 15580/01/NL/HB

[3] DeBra D B 1997 Class. Quantum Grav. 141549

[4] Smith G I et al 1999 Phys. Rev. D 61022001

[5] Vitale S et al 2002 Nucl. Phys. B 110210

[6] Weber W J et al 2002 Proc. SPIE Astronomical Telescopes and Instrumentation Conf.

[7] Dolesi R et al 2003 Proc. 4th Int. LISA Symp. (Pennsylvania, July 2002) Class. Quantum Grav. 20 S99

[8] Da Lio M and Vitale S 2001 Electrostatic suspensions for LISA and SMART ESTEC drag-free control contract $13691 / 99 / \mathrm{NL} / \mathrm{FM}$

[9] Heinzel G et al 2003 Proc. 4th Int. LISA Symp. (Pennsylvania, July 2002) Class. Quantum Grav. 20 S153

[10] Araújo H M, Howard A, Davidge D and Sumner T J 2002 Proc. SPIE Astronomical Telescopes and Instrumentation Conf.

[11] Araújo H M et al 2003 Proc. 4th Int. LISA Symp. (Pennsylvania, July 2002) Class. Quantum Grav. 20 S311

[12] Sandford M et al 2002 Talk presented at 4th Int. LISA Symp. (Pennsylvania, July 2002)

[13] Tirabassi C et al 2002 Talk presented at 4th Int. LISA Symp. (Pennsylvania, July 2002)

[14] Hanson J et al 2003 Proc. 4th Int. LISA Symp. (Pennsylvania, July 2002) Class. Quantum Grav. 20 S109 